Chirurg 2015 $\cdot 86: 376$

DOI 10.1007/s00104-015-3019-8

Online publiziert: 29. März 2015

๑) Springer-Verlag Berlin Heidelberg 2015

H. Dralle

Universitätsklinik für Allgemein-, Viszeral- und Gefäßchirurgie, Medizinische

Fakultät, Universitätsklinikum Halle (Saale), Halle (Saale)

\title{
Primärtumorresektion bei hepatisch metastasierten neuroendokrinen Pankreastumoren
}

der operierten (12) gegenüber den nichtoperierten Patienten (31).

\section{Ergebnisse}

tion of the primary pancreatic neuroendocrine tumor in patients with unresectable liver metastases: possible indications for a multimodal approach. Surgery 155:607-614

\section{Hintergrund und Fragestellung}

Lebermetastasen sind nicht selten die einzige systemische Manifestation neuroendokriner Pankreastumoren (PNET), eine radikale Lebermetastasenresektion ist jedoch nur bei einem kleinen Teil dieser Patienten möglich. Die Frage ist daher, ob PNET-Patienten mit primär synchronen, nicht radikal resektablen Lebermetastasen von einer Primärtumorresektion profitieren oder diese nur bei symptomatischen tumorbedingten Komplikationen (z. B. Blutung, Obstruktion) gerechtfertigt ist.

\section{Methoden}

Innerhalb eines retrospektiv 10-jährigen Untersuchungszeitraumes konnten insgesamt 43 Patienten mit synchroner und bildgebend ausschließlicher, nichtresektabler Lebermetastasierung in die Analyse einbezogen werden. 72 der 115 Patienten mit PNET wurden ausgeschlossen (keine Metastasen $n=60$; extrahepatische Metastasen $n=7$; resektable Lebermetastasen $n=5)$. Das tumorbefallene Lebervolumen wurde nach drei Kategorien beurteilt: $<25 \%, 25-50 \%$, $>50 \%$ des Lebergesamtvolumens. Ziel der Untersuchung war die uni- und multivariate Analyse des Gesamtüberlebens („overall survival“, OS)
Die Entscheidung über ein operatives vs. nichtoperatives Vorgehen bez. des Primärtumors wurde im multidisziplinären Tumorboard getroffen. Ausschlusskriterien für eine Operation waren eine Infiltration der Portalvene oder des Truncus coeliacus bzw. der A. mensentrica superior. 10 Patienten erhielten eine Pankreaslinksresektion, 2 eine Kopfresektion. Keiner der Patienten verstarb postoperativ. Bei allen 12 Patienten wurden initial und im weiteren Verlauf zusätzlich unterschiedliche Systemtherapien durchgeführt.

Das 5-Jahres-OS der operierten Patienten betrug $82 \%$, das der nichtoperierten $50 \%(\mathrm{p}=0,027)$. Tumorbezogene Einflussfaktoren auf das OS waren in der multivariaten Analyse die Primärtumorresektion, der Ki67 und das Tumorvolumen des Leberbefalls.

\section{Diskussion und Fazit}

Die vorliegende Untersuchung ist nach Einschätzung der Autoren die erste, die multivariat zeigen konnte, dass beim hepatisch metastasierten PNET die Primärtumorresektion mit einem etwa $30 \%$ besseren $\mathrm{OS}$ verbunden ist als eine ausschließlich nichtoperative multimodale Therapie. Auch die postoperative NullLetalität der vorliegenden Studie spricht dafür, dass das Tumorboard bei der Behandlungstriage die richtige Patientenselektion hinsichtlich eines operativen bzw. nichtoperativen Vorgehens getroffen hat.

Die meisten der in der vorliegenden Studie primärtumorresezierten Patienten hatten Grad 1 oder 2 bzw. Ki67<20\%-Tumoren. Trotz der Einschränkungen, die die vorliegende Untersuchung notwendigerweise hat (kleine Fallzahl, retrospektive Analyse, inhomogenes Patientengut $u$. a.) zeigen die Ergebnisse nicht nur, dass PNET auch günstigerer Kategorien hepatisch metastasieren können (und deshalb bestmöglichst vor erfolgter Metastasierung reseziert werden sollten), sondern auch, dass bei eingetretener Lebermetastasierung Primärtumorresektionen unter entsprechenden Voraussetzungen (Allgemeinzustand, Resektabilität) von Vorteil für die Überlebenschancen sind und darüber hinaus einen hepatisch gezielten Einsatz multimodaler Therapieverfahren ermöglichen [1].

\section{Korrespondenzadresse}

Prof. Dr. Dr. h.c. H. Dralle FRCS, FACS, FEBS Universitätsklinik für Allgemein-, Viszeral- und Gefäßchirurgie, Medizinische Fakultät, Universitätsklinikum Halle (Saale) Ernst-Grube-Str. 40, 06097 Halle (Saale) henning.dralle@uk-halle.de

Interessenkonflikt. H. Dralle gibt an, dass kein Interessenkonflikt besteht.

\section{Literatur}

1. Frilling A, Modlin IM, Kidd M et al (2014) Recommendations for management of patients with neuroendocrine liver metastases. Lancet 15:e8e21 